\title{
Supporting Information: Rational Design of Nile Red Analogs for Sensing in Membranes
}

\author{
Salvatore Prioli, Peter Reinholdt, Mick Hornum, and Jacob Kongsted* \\ Department of Physics, Chemistry and Pharmacy, University of Southern Denmark, \\ Campusvej 55, DK-5230 Odense M, Denmark \\ E-mail: kongsted@sdu.dk
}




\section{Nile red screening}

The RDKit script used in the generation of the Nile Red analogs is shown in listing 1.

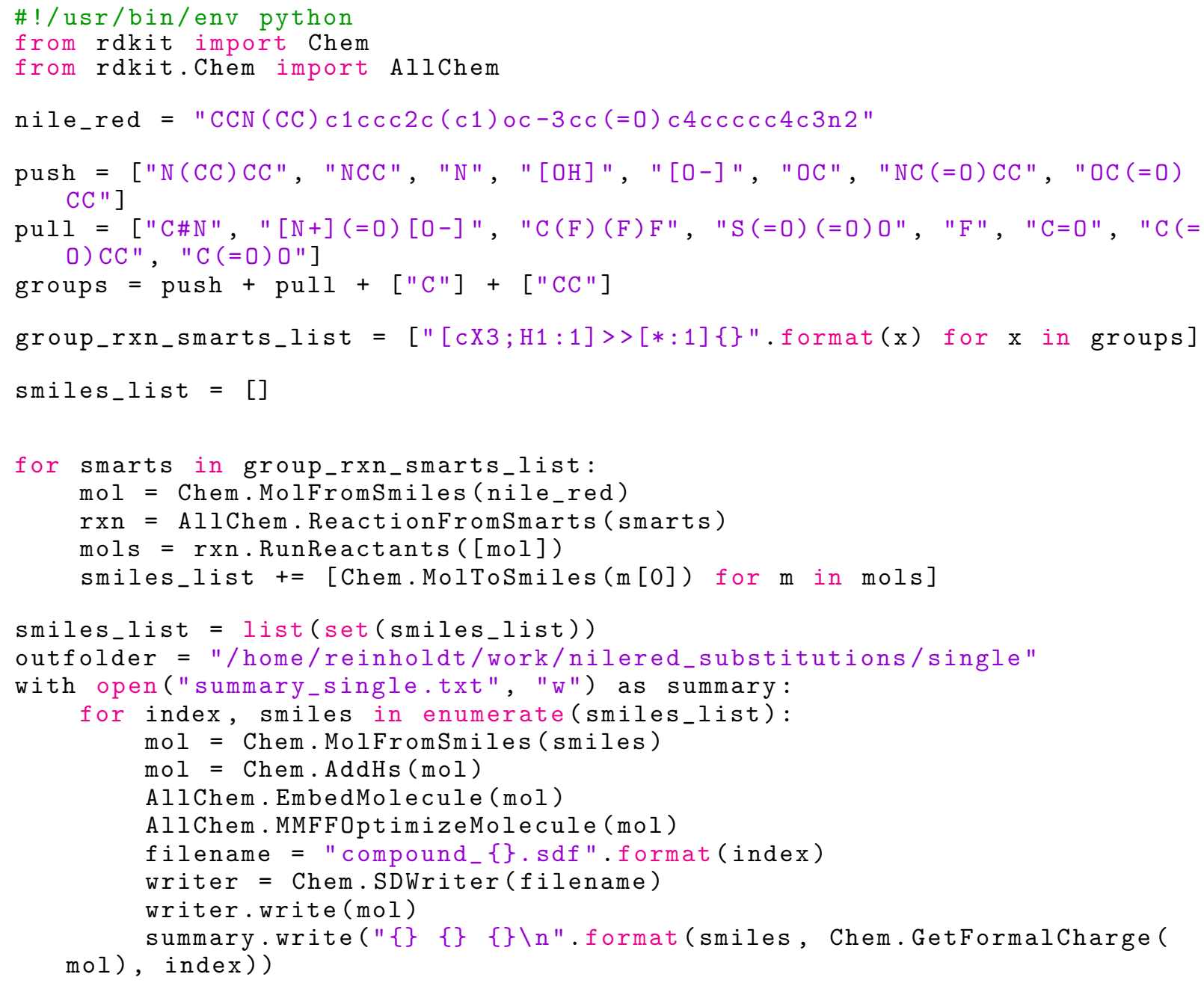

Listing 1: Nile red analog generation script

\section{The charge transfer mechanism: LE and TICT structures}

Nile Red consists of three subunits: the diethyl amino group which works as donor (D), the ketone which works as acceptor (A) and the $\pi$ framework linking these two subunits and to which the substituents are bonded. The photoexcitation induces a charge transfer from $\mathrm{D}$ to A (Figure 3b) and gives rise to the characteristic solvatochromism of Nile Red. ${ }^{1}$ However, it is not yet completely understood why Nile Red has a double fluorescence and through which mechanism this process occurs. ${ }^{2}$ Different hypotheses can be found in the literature but here 
- in order to explain in a qualitative way the different behaviour of the substituents - we will use the locally excited (LE) and twisted intramolecular charge transfer (TICT) structures hypothesis. ${ }^{3}$ Both conformations correspond to emissive states but differ in the dihedral angle of the diethyl amino group with the $\pi$ system plane. The LE has a structure which resembles closely the ground state (GS) equilibrium structure where the three subunits tend to assume a planar conformation in order to maximize the conjugation between A and D. Due to geometrical relaxation in the excited state, it is found that this structure presents a more decreased bond length alternation (BLA), i.e. increased double bond character, with respect to the ground state. So the enolate resonance structure dominates in the excited state with a partial positive charge on $\mathrm{D}$ and a partial negative charge on A. On the other hand, the TICT structure has a negative charge even more shifted towards the A subunit since the conjugation between $\mathrm{D}$ and $\mathrm{A}$ is blocked. The TICT structure appears after an adiabatic reaction on the $S_{1}$ potential energy surface.

TD-DFT methods can be used to describe the charge transfer (CT) mechanism but with an accuracy and reliability that strongly depends on the functional. ${ }^{4}$ It is known that the energy of the CT state can be overestimated by DFT depending on the weight of exact exchange in the functional which overstabilize the TICT structure. ${ }^{5}$ More accurate methods demonstrate that the TICT structure is more stable of $3 \mathrm{kcal} / \mathrm{mol}$ and the barrier is of about $5 \mathrm{kcal} / \mathrm{mol} .{ }^{6}$ With the DFT method used all the analog structures maintain the same Nile Red (NR) structure with the three subunits D- $\pi$-A highly conjugated with the $\pi$ framework. For analogs $\mathbf{1}, \mathbf{2}, \mathbf{3}, \mathbf{4}, \mathbf{5}, \mathbf{6}$ and $\mathbf{7}$ the spectroscopic behaviour can be associated with the LE structure mechanism. In the excited state optimized structure $\left(E S_{\text {opt }}\right)$ the BLA decreases by ca. $0.03 \AA$ while the dipole moment increase (Table S1). Based on the resonance structures (Figure 3b) the effect of the substituents can be partly explained. First, the small red-shift for analogs bearing fluorine. This is slightly higher for $\mathbf{1}$ and $\mathbf{2}$ compared to for $\mathbf{6}$ and $\mathbf{7}$. The effect of fluorine is basically to stabilize the resonance structure with the charge separated in the GS and in the ES optimized structures so the energy of the absorption and emission is 
decreased with respect to NR. Analogs $\mathbf{3 , 4}$ and $\mathbf{5}$ have substituents that can conjugate with the $\pi$ system and therefore a red-shift is expected. For example analog $\mathbf{5}$ has red-shifted absorption and emission with respect to $\mathbf{N R}$ since the methoxy group is aligned with the conjugated framework increasing the dimension of the $\pi$ system. However, due to steric clashes, analog $\mathbf{3}$ has not the substituent aligned with the $\pi$ system as can be seen in Figure S1 meaning that it is less red-shifted than $\mathbf{5}$.

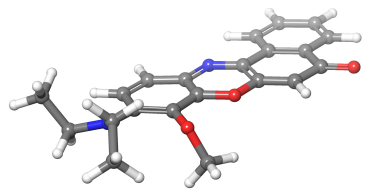

(a)

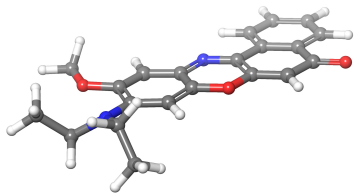

(b)

Figure S1: Ground state optimized structure of analog 3 (a) and analog $\mathbf{5}$ (b). The different conformation of the methoxy substituent can here be seen.

In analog 8 an additional diethyl amino group has been introduced close to the donor part of the NR $\pi$ subunit. The structure of this analog is completely different with respect to NR and all the other analogs. The introduction of the additional diethyl amino group forces the original one to assume a twisted position (see Figure S2). In this way high redshifted absorption and emission is observed with high dipole moment variation between the ground and excited state optimized structure (Table S2) but with a reduced BLA variation. This is because analog 8 assumes a TICT structure already in the GS optimized state which correspond to a higher energetic point on the ground state PES and lower in the excited state compared to NR. Thus, this is why a big red-shift is observed in absorption and emission. 


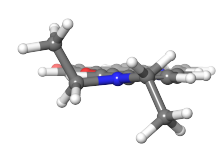

(a)

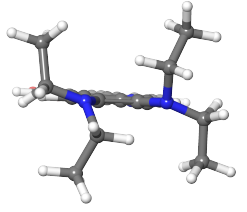

(b)

Figure S2: Ground state optimized structures of NR (a) and analog 8 (b). The analog 8 assume a TICT structure already in the ground state. The same structure can be assumed by $\mathbf{N R}$ by overcoming a barrier in the excited state while $\mathbf{8}$ is forced to this structure by the presence of the substituent.
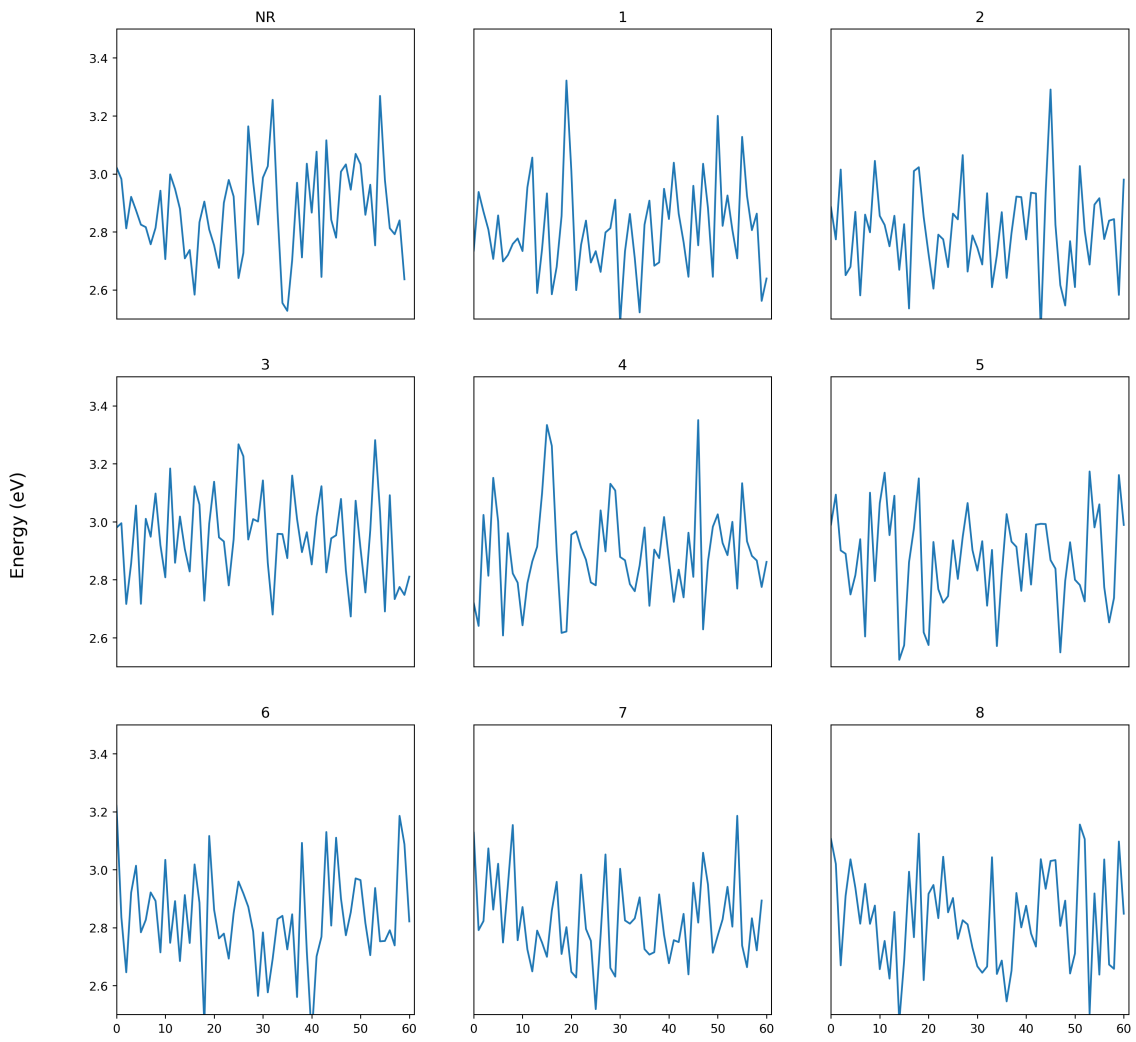

Snapshot

Figure S3: Excitation energy calculated using MM-sampled structures within the membrane environemnt. The excitation energy (in $\mathrm{eV}$ ) is reported as a function of snapshot number. 
Table S1: Dipole moments for NR and the considered analogs for the ground state optimized structure (GS), structure obtained after vertical excitation (ES) and optimized structure in the excited state $\left(\mathrm{ES}_{\text {opt }}\right)$. The difference between them is also reported. All the reported values are in Debye (D).

\begin{tabular}{ccccccc} 
& \multicolumn{3}{c}{$\mu$} & & & \multicolumn{2}{l}{$\Delta \mu$} \\
\cline { 2 - 3 } \cline { 6 - 7 } Probe & GS & ES & ES $_{\text {opt }}$ & & ES-GS & ES-ES opt \\
\hline NR & 9.20 & 12.98 & 11.95 & & 3.92 & 2.88 \\
$\mathbf{1}$ & 12.68 & 16.49 & 15.32 & & 4.03 & 2.87 \\
$\mathbf{2}$ & 11.09 & 15.07 & 14.00 & & 3.98 & 2.91 \\
$\mathbf{3}$ & 8.06 & 12.41 & 11.36 & & 4.56 & 3.50 \\
$\mathbf{4}$ & 10.61 & 14.11 & 13.22 & & 3.61 & 2.68 \\
$\mathbf{5}$ & 9.12 & 12.74 & 12.01 & & 3.93 & 3.18 \\
$\mathbf{6}$ & 11.08 & 15.05 & 13.97 & & 4.03 & 2.95 \\
$\mathbf{7}$ & 9.93 & 13.76 & 12.69 & & 3.87 & 2.82 \\
$\mathbf{8}$ & 8.35 & 13.41 & 14.25 & & 5.29 & 6.02 \\
\hline
\end{tabular}
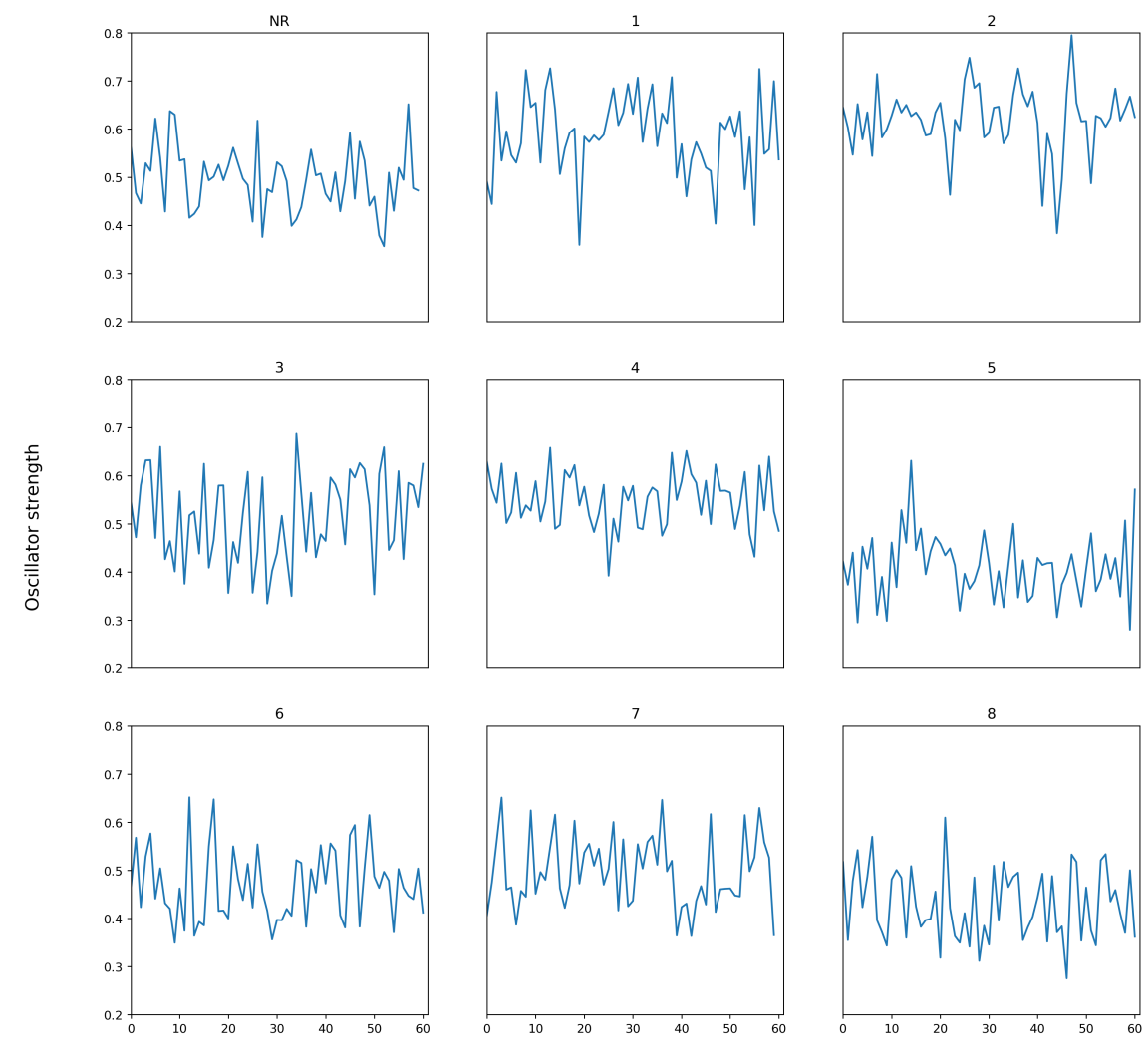

Snapshot

Figure S4: One photon absorption intensity (oscillator strength) calculated using MMsampled structures as a function of snapshot number within the membrane environment. 
Table S2: Bond length alternation (BLA) in $\AA$ calculated for all the probes in the ground and excited state optimized structures. The BLA has been obtained as the difference between the average single and and double bonds lengths. The last column shows the change in BLA between the grond and excited states.

\begin{tabular}{cccc} 
Probe & GS & $\mathrm{ES}_{\text {opt }}$ & $\Delta$ \\
\hline NR & 0.064 & 0.029 & 0.035 \\
$\mathbf{1}$ & 0.064 & 0.031 & 0.033 \\
$\mathbf{2}$ & 0.061 & 0.030 & 0.032 \\
$\mathbf{3}$ & 0.068 & 0.032 & 0.036 \\
$\mathbf{4}$ & 0.063 & 0.026 & 0.037 \\
$\mathbf{5}$ & 0.061 & 0.034 & 0.027 \\
$\mathbf{6}$ & 0.063 & 0.030 & 0.034 \\
$\mathbf{7}$ & 0.064 & 0.029 & 0.034 \\
$\mathbf{8}$ & 0.071 & 0.055 & 0.015 \\
\hline
\end{tabular}
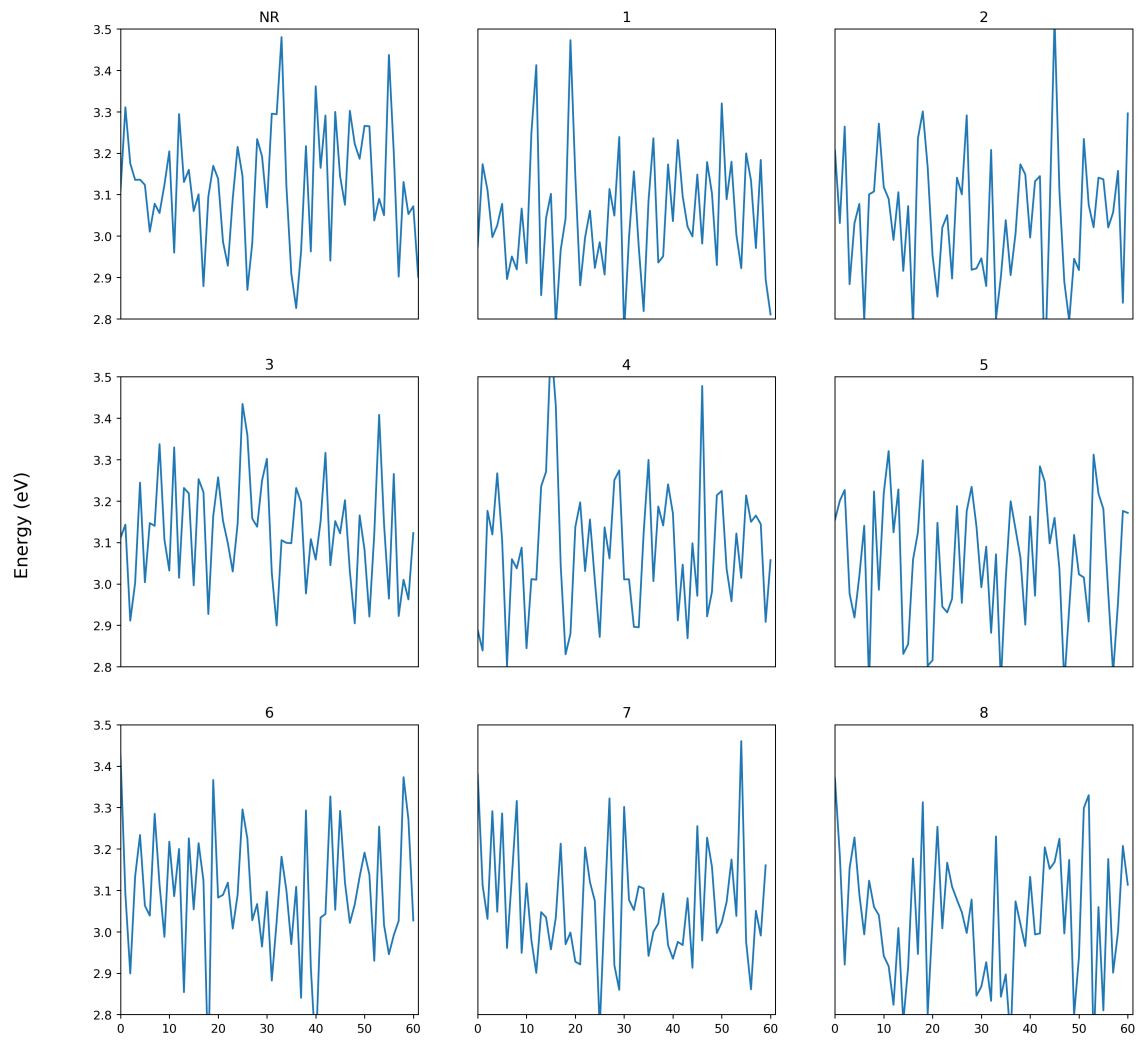

Snapshot

Figure S5: Excitation energy calculated using MM sampled structures in gas-phase. The excitation energy (in $\mathrm{eV}$ ) is reported as a function of snapshot number. 

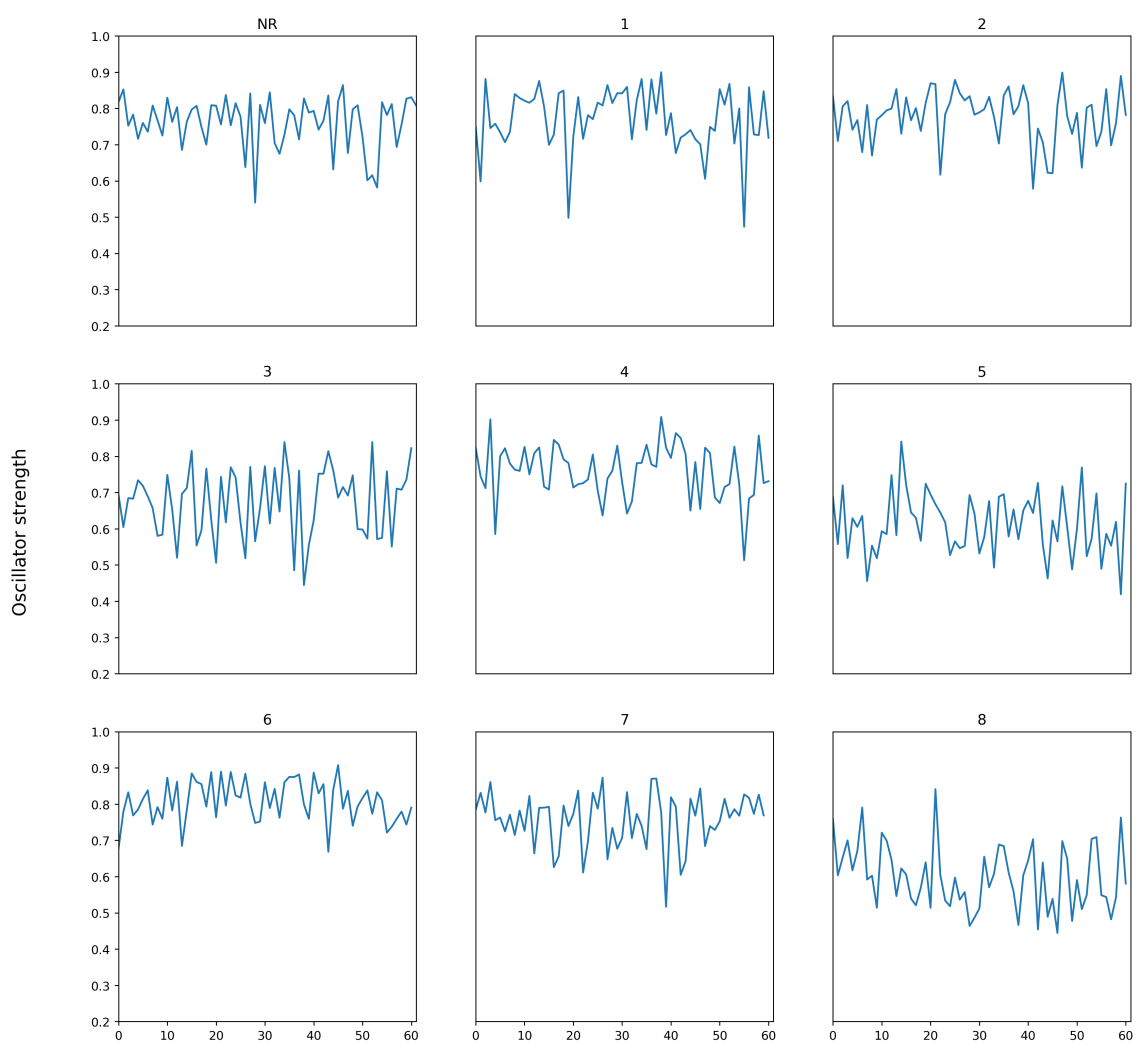

Snapshot

Figure S6: One photon absorption intensity (oscillator strength) calculated using MMsampled structures as a function of snapshot number in gas-phase.

Table S3: The lowest excitation energy (in $\mathrm{eV}$ and $\mathrm{nm}$ ) together with the oscillator strength calculated for the MM-sampled structure in gas-phase.

\begin{tabular}{ccc} 
& \multicolumn{2}{c}{ MMs } \\
\cline { 2 - 3 } Probe & $\Delta \mathrm{E}$ & $f$ \\
\hline $\mathbf{N R}$ & $3.12(397)$ & 0.76 \\
$\mathbf{1}$ & $3.05(407)$ & 0.77 \\
$\mathbf{2}$ & $3.05(407)$ & 0.78 \\
$\mathbf{3}$ & $3.13(397)$ & 0.67 \\
$\mathbf{4}$ & $3.08(402)$ & 0.76 \\
$\mathbf{5}$ & $3.06(405)$ & 0.61 \\
$\mathbf{6}$ & $3.09(401)$ & 0.81 \\
$\mathbf{7}$ & $3.06(405)$ & 0.76 \\
$\mathbf{8}$ & $3.04(408)$ & 0.60 \\
\hline
\end{tabular}




\section{References}

(1) Dutta, A. K.; Kamada, K.; Ohta, K. Spectroscopic Studies of Nile Red in Organic Solvents and Polymers. J. Photochem. Photobiol. 1996, 93, 57-64.

(2) Dutt, G.; Doraiswamy, S.; Periasamy, N. Molecular Reorientation Dynamics of Polar Dye Probes in Tertiary-butyl Alcohol-water Mixtures. J. Chem. Phys. 1991, 94, 5360-5368.

(3) Grabowski, Z. R.; Rotkiewicz, K.; Rettig, W. Structural Changes Accompanying Intramolecular Electron Transfer: Focus on Twisted Intramolecular Charge-transfer States and Structures. Chem. Rev. 2003, 103, 3899-4032.

(4) Tuck, P. O.; Mawhinney, R. C.; Rappon, M. An Ab Initio and Td-dft Study of Solvent Effect Contributions to the Electronic Spectrum of Nile Red. Phys. Chem. Chem. Phys. 2009, 11, 4471-4480.

(5) Dreuw, A.; Head-Gordon, M. Single-reference Ab Initio Methods for the Calculation of Excited States of Large Molecules. Chem. Rev. 2005, 105, 4009-4037.

(6) Ya. Freidzon, A.; Safonov, A. A.; Bagaturyants, A. A.; Alfimov, M. V. Solvatofluorochromism and Twisted Intramolecular Charge-transfer State of the Nile Red Dye. Int. J. Quantum Chem. 2012, 112, 3059-3067. 